\title{
Differences in Survivability among F344 Rats
}

\author{
Shin TANAKA ${ }^{1)}$, Norika TAMAYA ${ }^{1)}$, Kaori MATSUZAWA2), \\ and Osamu MIYAISHI2)
}

\author{
${ }^{1)}$ Laboratory Animal Research Facilities (LARF) and ${ }^{2)}$ Laboratory of Pathology, \\ Department of Basic Gerontology of National Institute for Longevity Sciences (NILS), \\ 36-3, Gengo Morioka-cho, Obu 474-8522, Japan
}

\begin{abstract}
Important parameters to identify and develop appropriate animal models for longevity science include survivability, age-related disorders, and easy handling of aged individuals. It is found that F334/Du and F344/N have distinctive strain difference in these parameters. The finding suggests F334/Du and F344/N, even though they are historically siblings, need clearly separate identification when used as animal models for aging science, in particular, longevity science.
\end{abstract}

Key words: aging model, F344/N and F344/Du, strain difference, survivability

Laboratory strains of small rodents, mice and rats have been established as animal models for biomedical science with detailed controls over factors related to genetics, microbiology and nutrition but not necessarily specific time that has lapsed. In aging science, it is aged/aging laboratory rodents that are essential for understanding the mechanisms of aging at the individual level.

When aged/aging laboratory rodents were not commercially available, some groups of researchers on aging in Japan and US, including the National Institute on Aging (NIA), Maryland, tried to establish aging farms (A/F) to produce colonies of rodents for their research. NIA developed A/Fs under both private and contractual arrangement with funding by the National Institute of Health (NIH), Maryland (See the latest NIA catalogue for animal resources).

Longevity science pursues quality of life for human beings. It mainly consists of basic and social gerontol- ogy and geriatrics. Basic gerontology requires animal models that are aged in good health without substantial disorders, while geriatrics needs those that significantly develop age-related disorders. It is therefore important to identify and develop effective parameters for animal models to meet specific needs in basic gerontology and geriatrics. The parameters should also be defined sensitively by incorporating time and individuals.

In aging science a uniquely significant parameter, survivability (SvA), has been developed for laboratory rodents. SvA is the accumulated life span of individual rodents. If a factor that may modify SvA can be pathologically identified as an disorder, it will help to improve the quality of laboratory rodents as animal models, thus contributing to geriatric research. It also means that after such a disorder modifies SvA, the handling or maintenance of animals will become difficult, since SvA, disorders and ease of handling are closely related to one another, and are easy to make estimations exter-

(Received 12 July 1999 / Accepted 3 February 2000)

Address corresponding: S. Tanaka, Laboratory Animal Research Facilities (LARF) of National Institute for Longevity Sciences (NILS), 36-3, Gengo Morioka-cho, Obu 474-8522, Japan 
nally. They therefore seem to be very effective parameters and, thus, it is convenient to use them as parameters to assess, compare and establish appropriate animal models.

The 22nd Annual Meeting of the Japan Society for Biomedical Gerontology (JSBG) was held at Kyoto International Conference Hall from June 16 to 18, 1999. Among reports presented during the meeting were those, which dealt with "F344" rats, which seem to pose interesting discrepancies when collectively analyzed. Such differences can be categorized into two groups, the one related to biological characteristics of "F344" rats and the other attributable to nonspecific notation or description of strains. This report aims to clarify such discrepancies with attentions to differences among substrains.

In the first group, notable discrepancies are related to biological characteristics in view of the aforementioned three parameters; SvA, disorders and ease of handling.

The first discrepancy is found in the half life span or $50 \%$ SvA of male rodents as compared in Table 1. The half life span of "F344" was 29 months of age in the report by Toho University (Toho) [5], whereas 50\% SvA was 24.8 months for "F344/N" at the National Institute for Longevity Sciences (NILS) [8].

The second discrepancy concerns age-related disorders. NILS reported that hematological disorders, including both leukemia and anemia, were the highest at $84.70 \%$ for "F344/N" [4]. The Tokyo Metropolitan Institute of Gerontology (TMIG) reported that the incidence of leukemia was moderate (about 50\%) for "F344/ DuCrj"[7].

The third discrepancy is related to general ease of handling as laboratory rodents. NILS reported that "F344 " and "F344/N" were very difficult to use [3, 4, 8], but Nagasaki University (Nagasaki) did not include "F344" in the same category [6].

These discrepancies among the four institutes, as contrasted by three parameters, are too significant to ignore. It may even be hard to accept them as the results of observing the same strain of rats. "F344 rats" have been recommended as a suitable laboratory strain for aging science partly because of their advantages in terms of long-term breeding and small body size. Nevertheless, reported "F344" rats are not consistent enough in biological characteristics, especially the long median life span (31 months for males and 29 months for females) and the incidence of various tumors [2] (Table 1). They were mononuclear cell leukemia ( $24 \%$ for males), mammary tumors (41\% for females and $23 \%$ for males), pituitary adenomas (36\% for females and $24 \%$ for males), testicular interstitial cell tumors ( $85 \%$ for males) [2]. In particular the reported incidence of leukemia and pituitary adenomas is obviously different from what has been observed among "F344/N" at NILS [2] (Table 1).

Because of these inconsistent biological characteristics, it is not appropriate to recommend "F344" rats, with a higher incidence of leukemia, as animal model for longevity science.

In the second group, differences are related to the way "F344" rats are identified. At the JSBG meeting, out of sixty-six reports registered for the general oral presentation session (one of them was canceled later), forty reports dealt with laboratory strains of rodents in their research. As shown in Table 2, laboratory strains of rats reported at the meeting can be divided into four

Table 1. Major Differences Reported on Male F344

\begin{tabular}{|c|c|c|c|c|}
\hline & & & & Reference [2] \\
\hline Half Life Span & 29 Months & & & 31 Months \\
\hline Fifty percent Survival & Toho & vs & $\begin{array}{l}24.8 \text { Months } \\
\text { NILS }\end{array}$ & \\
\hline $\begin{array}{l}\text { Pathology with Aging } \\
\text { (Leukemia Incidence) }\end{array}$ & $\begin{array}{l}\text { Moderate } \\
50 \% \\
\text { TMIG }\end{array}$ & vs & $\begin{array}{l}\text { High } \\
84.6: \% \\
\text { NILS }\end{array}$ & $\begin{array}{l}\text { Low } \\
24 \%\end{array}$ \\
\hline General Availability & $\begin{array}{l}\text { Good } \\
\text { Nagasaki }\end{array}$ & vs & $\begin{array}{l}\text { Not so good } \\
\text { NILS }\end{array}$ & Good \\
\hline
\end{tabular}


Table 2. Laboratory Strains of Rodents Used at the 22nd Annual Meeting of Japan Society for Biomedical Gerontology

\begin{tabular}{llll}
\hline Order & Species & Strain & \\
\hline Rodentia & Rats (22)* & & (Description of strain) \\
& & Wistar (4) & (Wistar) \\
& Donryu (1) & (Donryu) \\
Unidentified & (Not described) \\
& & Others (16) & (F344, F-344, F344/N purchased from SLC, F344/DuCrj, \\
& & Fischer 344 rat and Fischer) \\
& & \\
& & \\
& & SAM (13) & \\
& & C57BL/6 (18) \\
& Others (1) & \\
\hline
\end{tabular}

*Numbers of representations are shown in parenthesis.

categories: Wistar (4 reports), Donryu (1), others (16) and unidentifiable (1) (Table 2). Others include both explicitly named "F344" such as F344/DuCrj or F344/ $\mathrm{N}$ and ambiguously identified "F344" such as F-344, Fischer 344 rats, Fischer or F344. The description used by Toho, Nagasaki, and NILS is grouped into "others".

Precise strain names used by Toho and Nagasaki became available through our interviews with their researchers. They said their "F344" rats were purchased from Charles River Japan (CRJ), Yokohama. This indicates that their rats are F344/DuCrj, the same as those used by TMIG.

Biological characteristics common among inbred strains are generally stable and constant, although there are many factors that may affect or modify the characteristics. In the abovementioned case, there are marked differences in biological characteristic between NILS on one side and Toho, Nagasaki and TMIG on the other, as shown in Table 1. With precise notation, the strain used by NILS is identifiable as F344/N while that used by Toho, Nagasaki and TMIG is identifiable as F344/ Du. By taking into consideration these two differences, i.e. in biological characteristics and strain notation, one can suggest that inconsistency in biological characteristic among Toho, TMIG, Nagasaki and NILS may reflect indigenous differences between F344/Du purchased from CRJ and F344/N from SLC Ltd. (SLC), Hamamatsu, resulting from differences by strain.

To make this point clear, we tried to trace the history of "F344" rats to understand the differences in biological parameters summarized in Table 1 and to clarify differences in the notation of strain names as listed in
Table 2.

"F344" stemmed from a colony established at the Institute for Cancer Research, Columbia University (CU) under the Columbia Project in 1919 aiming at the development of rat models for cancer research at $\mathrm{CU}$ (Fig. 1). Drs. Curtis, Dunning and Bullock promoted this project at $\mathrm{CU}$. $\mathrm{CU}$ purchased the first rat stocks from seven different breeders, including Fischer, August, Marshall and Zimmerman, and a stock from Copenhagen, Denmark. Subsequent inbreeding led to the development of various inbred strains [1]. One of such inbred strains was F344 [2] and it had been characterized as mentioned above. This originated in the three hundred and forty fourth pair of Fischer and was named F344.

In 1949, some F344 rats were shared with Dr. Heston at the National Cancer Institute, NIH. In the next year, he added /N to F344 on F51 to identify the shared F344 as a sub-line at NIH, from an original colony at CU (Fig. 1). Based on descendants of this F344/N, NIA established a contract A/F at Harlan Sprague Dewley (HSD), Indiana [3] (Fig. 1), so that F344/N rats now available from contract $\mathrm{A} / \mathrm{F}$ at HSD through NIA should be described as F344/NHsd. Although HSD adopted this name, NIA introduced this strain in the catalogue for animal resources as Fischer 344.

SLC introduced breeding nuclei of F344/N from the Institute of Medical Science, University of Tokyo (IMSUT) on F138 in 1980 (Fig. 1). SLC established a Specific Pathogen Free (SPF) colony of F344/N through Caesarean section after breeding them. SPF F344/N therefore became available commercially as F344/NSlc. 


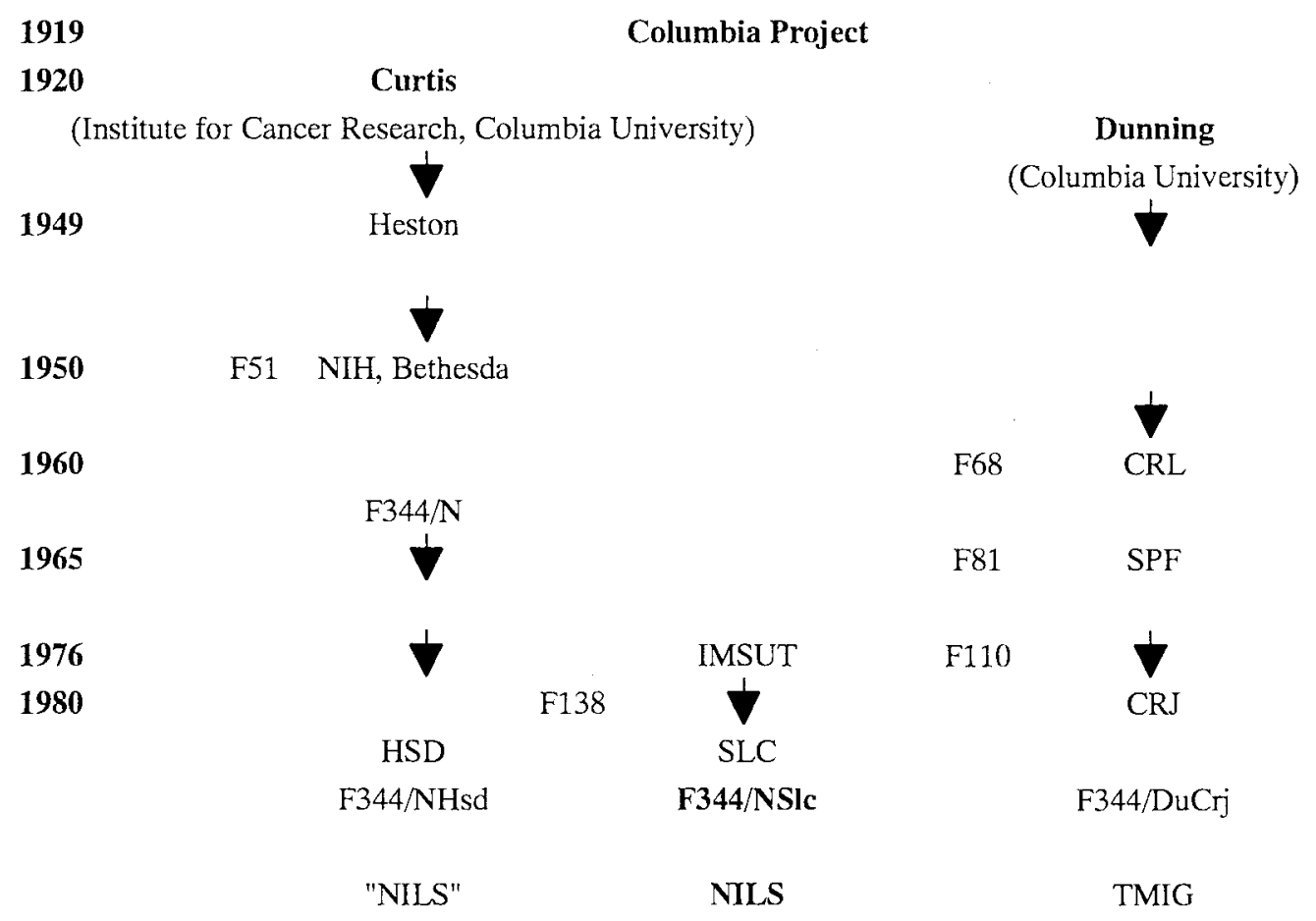

Fig. 1. Breif History of F344/N and F344/Du.

NILS established its own A/F on this F344/NSlc. At the current meeting NILS reported its findings on this F344/N [4, 8] or F344/NHsd purchased from HSD [3].

Along an original line of F344, there is another major strain. Charles River Laboratories (CRL) introduced breeding nuclei of F344 rats from Dr. Dunning on F68 in 1960, 11 years after the sharing with Dr Heston, and named F344/Du to distinguish it from CU colony. In 1965, CRL established SPF colony through Caesarean section of this strain on F81. CRJ introduced a descendant of this SPF F344/Du on F110 in 1976. As a result, F344/Du became available commercially as F344/DuCrj in Japan.

By tracing the history, it became clear that there are three major strains for "F344" rats: the original F344 at CU, F344/N sub-lined at NIH and F344/Du sub-lined at CRL. A half a century has passed since the segregation of F344/N from the original F344, and forty years since the segregation of F344/Du. It should be noted that even among inbred strains, segregation for several decades may be enough to differentiate them one from another in terms of their biological characteristics.

In Japan, only two sub-lined strains, but original F344 have been available. They can be traced back by pedigree to either the sub-lined strain at NIH (F344/N) distributed from SLC, or the Dunning strain of CRL (F344/Du) from CRJ [2].

In the JSBG case, therefore, inconsistent biological characteristics associated with "F344" rats can be explained as strain differences between F344/Du and F344/N with the help of detailed notation of their names and confirmation of their history. In fact, this report is intended for the first time to suggest strain related difference between the siblings, F344/Du and F344/N. It should be more appropriate to regard the two siblings as separate identities in terms of biological characteristics, in particular, that are important to researches of longevity science. Researchers should be alerted to direct their attention to a specific strain, F344, Dunning or NIH. 
For conventional usage other than aging science, it is also recommended to distinguish those characteristics that are common among "F344" rats (including testicular interstitial cell tumor $[2,4])$ and those that are specific either to F344/N (shorter life span [8], early onset of severe leukemia and sex difference in pituitary adenoma [4]) or to F344/Du (longer life span [5] and low incidence of leukemia [7]).

F344/Du rats appear to be more appropriate as animal models for general aging and longevity researches because their longer life span and lower incidence of tumor when compared to F344/N. Since most laboratory rodent strains have been developed for oncology, F344/N might have been bred with an inclination to a higher incidence of leukemia or accumulation of mutations modifying the incidence. This makes F344/N rats more suitable animal models for oncology and geriatrics than longevity science, although age-related hematological disorders that are characteristic to F344/ $\mathrm{N}$ are not so common among aged human beings.

The differences related to the reports in the JSBG meeting represent phenotypic differences between sibling strains. They indicate two points: that identifiable genetic differences may exist between the two strains, and that both the strains may have a shorter life span than expected as a result of a higher incidence of leukemia. In other words, F344/Du rats may tilt towards F344/N in the characteristic equation, and F344/N may go further. For this reason, the sibling strains require other genetic methods for strict comparison in order to identify respective loci responsible for strain differences in leukemia incidence.

When researchers precisely describe the names of strains they use, they can eliminate confusing description such as F344, F-344, Fischer 344 and Fischer (Table 2), and avoid issues resulting from such imprecise descriptions. Furthermore, they can easily refer to and obtain information accumulated on the respective strains.

Some institutes, including NIA in the US, have recently faced problems related to the biological characteristics of "F344". They include the shortening of the half life span (NIA) and the increase in the incidence of leukemia with splenomegaly (Tufts University, Massachusetts). Strain differences between F344/Du and F344/N together with clear identification of strain names can serve as a key to solve these problems.

\section{Acknowledgments}

This report was supported in part by funds from the Ministry of Health and Welfare (H10-Chouju-118) and National Chubu Hospital (H10-Itaku-04).

Authors wish to express their thanks to Dr. Kenichi KITANI, Director General of NILS, for his decision to establish an F344/N Aging Farm at NILS, and Drs. Sataro GOTO and Ryoya TAKAHASHI, of Toho University, Drs. Isao SHIMOKAWA and Yoshikazu HIGAMI, of Nagasaki University and Dr. Seigo SHUMIYA, of TMIG for their invaluable advice and kind answers to our questions.

\section{References}

1. Festing, M.F.W. 1979. pp. 234-235. In: Biological Handbooks III, Inbred and Genetically Defined Strain of Laboratory Animals. Part 1 Mouse and Rat (Altman, P.L. and Katz, D.D. eds.), Federation of American Societies for Experimental Biology, Bethesda.

2. Festing, M.F.W. 1979. pp. 277-278. In: Inbred Strains in Biomedical Research, Festing (M.F.W. ed.), The MaCmillan Press Ltd., London and Basingstoke.

3. Kitani, K., Minami, C., Maruyama, W., Yamamoto, T., Ohhashi, K., Naoi, M., Kanai, S., and Carrillo, M.C. 1999. Biomedical Gerontology 23: 31.

4. Miyaishi, O., Matsuzawa, K., Isobe, K., and Tanaka, S. 1999. Biomedical Gerontology 23: 32.

5. Nishizawa, K., Takahashi, R., and Goto, S. 1999. Biomedical Gerontology 23: 61.

6. Shimokawa, I., Ohta, K., Higami, Y., Tanaka, K., Tsuchiya, S., and Ikeda, T. 1999. Biomedical Gerontology 23: 60.

7. Shumiya, S., Kuramoto, K., Waki, H., Tanaka, Y., Ando, S., Itoh, H., and Kaneko, M. 1999. Biomedical Gerontology 23: 34 .

8. Tanaka, S., Segawa, T., Tamaya, N., and Miyaishi, O. 1999. Biomedical Gerontology 23: 32. 